J. Egypt. Soc. Parasitol. (JESP), 46(2), 2016: 253- 260

\title{
IMMUNE-MOLECULAR IDENTIFICATION OF GIARDIA INTESTINALIS IN DIARRHOEAL CHILDREN: COMPARISON OF THREE DIAGNOSTIC METHODS
}

\author{
By \\ NEVEEN A. MADBOULY ${ }^{1 *}$, ALYA FARID $^{1}$, AYMAN A. EL-BADRY $^{2}$ \\ and AZZA M. EL-AMIR ${ }^{1}$ \\ Department of Zoology ${ }^{1}$, Faculty of Science, Cairo University, Giza 12613, and De- \\ partment of Medical Parasitology ${ }^{2}$, Faculty of Medicine, Cairo University, Cairo, \\ Egypt ( ${ }^{*}$ Correspondence: aneveen@sci.cu.edu.eg)
}

\begin{abstract}
Giardiasis is a major health problem in both developed and developing world. A variety of methods for diagnosis of Giardia duodenalis cysts or trophozoites is available but still has certain limitations. 100 sample from diarrhoeal children who attending outpatient clinic in Abu El Rish hospital, Kasr Al Ainy, Faculty of Medicine, Cairo University, Egypt. Giardiasis was diagnosed by direct wet mount, microscopy after formal- ethyl acetate concentration, Ridascreen ELISA assay and n-PCR targeting beta giardin $(b g)$ gene. Using ELISA as reference standard, the methods' sensitivities, specificities, positive (PPV) and negative (NPV) predictive values and positive (LR + ) and negative (LR-) likelihood ratios with $95 \%$ confidence interval $(95 \% \mathrm{CI})$ were analyzed.The diagnostic methods were evaluated to determine their impact on the posttest probability using Fagan's nomogram. All the studied methods led to a LR+ higher than 10 indicating ability to ruling in giardiasis. n-PCR recorded LR- equal 0.00 and the probability of giardiasis would be $0 \%$ if the test was negative. The methods were also ranked on basis of Multiattribute utility theory and Analytical hierarchy process with ELISA ranked better than n-PCR.

Keywords: Giardia, Copro-DNA, bg gene, Ridascreen ELISA, Fagan's nomogram
\end{abstract}

\section{Introduction}

Giardia (G.) intestinalis stands out as the most frequent entero-parasite found in copro-parasitological surveys conducted in developed and developing countries. Giardia is not a life-threatening parasite; nevertheless, it gives rise to such problems as malabsorption and weight loss, leading to delayed growth and development in diarrheal children (Savioli et al, 2006). Giardia infection is usually diagnosed under light microscopy to identify either trophozoites or cysts in feces samples. Stool examination can be unreliable, as organisms may be excreted at irregular intervals which can produce a false negative test result (Adam, 2003). So that Enzyme immunoassays (EIAs) for detection of Giardia antigens in stool have replaced microscopy as the routine diagnostic procedure of choice in many hospitals and public health laboratories worldwide (Garcia and Shimizu, 1997). The reported sensitivities of EIAs ranged from 94 to $97 \%$ and specificities range from 99 to $100 \%$ (Johnston et al, 2003).

As a promising tool, polymerase chain reaction (PCR)-based techniques for diagnosis of giardiasis have been developed by many studies (El-Badry et al, 2010). However the role of PCR assay of copro-DNA remains controversial, especially to patients with positive PCR assays and negative microscopic results.

This study was designed to evaluate the diagnostic accuracy of n-PCR of coproDNA for diagnosis of giardiasis. Here nPCR based assay was used to amplify and detect a segment of beta giardin (bg) gene of G. intestinalis and was compared to conventional microscopy and commercially available Ridascreen ELISA kit for diagnosis of giardiasis in clinical stool specimens from children in Cairo, Egypt.

Subjects, Materials and Methods

A total of 100 stool samples were collected from diarrhoeal children ( 0 to 12 years old) who attending outpatient clinic in Abu El Rish Hospital, Cairo University, Egypt. 
The study was approved from the ethical committee in Parasitology department and the committee of the Faculty of Medicine, Cairo University, Egypt. Informed consent was obtained from the parents of all children participants included in the study.

All collected samples were examined microscopically with iodine wet mount, after formal- ethyl acetate concentration, immuno- and molecular studies. Coproscopy and immunoassay were carried out in the Diagnostic and Research of Parasitic Diseases (DRUP) and copro-PCR assay was held in the Lab of Molecular Medical Parasitology (LMMP), Department of Medical Parasitology, Faculty of Medicine, Cairo University, Egypt.

Microscopic examination: Stool samples were examined microscopically by direct and concentration methods for presence of Giardia trophozoite and cyst stages. Preparation was done using Lugol's iodine 5\% (Garcia, 2007).

Antigen detection using Ridascreen Giardia enzyme immunoassay (ELISA): Stool antigen detection was performed with Ridascreen Giardia enzyme immunoassay (ELISA) test (R-Biopharm AG, Landwehrstr, Darmstadt) according to the manufacturer's instructions. One positive and two negative controls were used at each run. A positive result was defined as an OD reading $10 \%$ over the cut-off value (negative control OD + 0.15), according to the manufacturer's instructions.

Polymerase Chain Reaction (PCR) assays: To optimize disruption of the cysts, prior to DNA extraction, the samples were subjected to five cycles of freezing and thawing by the following steps: two cycles alternating incubation in liquid nitrogen for $5 \mathrm{~min}$. and thawing in a water bath at $95^{\circ} \mathrm{C}$ for $5 \mathrm{~min}$. and concluding with a cycle of freezing in liquid nitrogen for $5 \mathrm{~min}$. and thawing at $95^{\circ} \mathrm{C}$ for $5 \mathrm{~min}$. Total DNA was extracted from all samples using Favorprep stool DNA isolation Mini Kit (Favorgen Biotech corporation Ping-Tung 908, Tai- wan, Cat. No.: FASTI001-1) following the manufacturer's instructions with modification in the form of prolongation of incubation to $95^{\circ} \mathrm{C}$ for $1 \mathrm{hr}$.

PCR Amplification: Molecular diagnosis of Giardia was done using beta-giardin (bg) gene. The extracted DNA was submitted to a nested procedure for amplification of a 511-bp region from the $b g$ gene (Caccio`et al, 2002). A nested- PCR protocol was used utilizing initial primers G7 (5'AAG CCC GAC GAC CTC ACC CGC AGT GC-3') and G759 (5'-CAT AAC GAC GCC ATC GCG GCT CTC AGG AA-3') amplifying a 753 bp product and secondary primers $\mathrm{F}$ (5'- GAA CGA ACG AGA TCG AGG TCC G-3') and R (5'CTC GAC GAG CTT CGT GTT-3`) generating a $511 \mathrm{bp}$ fragment. Amplification reactions were performed in a volume of 25 $\mu \mathrm{l}$ containing $3 \mu \mathrm{l}$ of DNA extract for first PCR reaction or $1 \mu \mathrm{l}$ of the second PCR reaction of the nested PCR, 12.5 $\mu$ l Qiagen master mix PCR kit (thermo scientific, U.K, Lot no.\#K1081), $1 \mu$ of each forward and reverse primer, $0.1 \mu$ of Taq polymerase

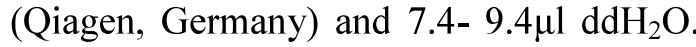
PCR reactions were performed in Biometra thermal cycler (Tpersonal). The PCR amplification reactions for Giardia were performed in condition consisted of $10 \mathrm{~min}$ at $95^{\circ} \mathrm{C}$ followed by 35 cycles of $30 \mathrm{~s}$ at $95^{\circ} \mathrm{C}$, $30 \mathrm{~s}$ at $65^{\circ} \mathrm{C}, 60 \mathrm{~s}$ at $72^{\circ} \mathrm{C}$ and then $7 \mathrm{~min}$ at $72^{\circ} \mathrm{C}$. Negative control reaction mixtures contained sterile distilled water in place of template DNA. Amplified DNA fragments were analyzed by electrophoresis in a $1.5 \%$ $(\mathrm{w} / \mathrm{v})$ agarose gel stained with ethidium bromide $(0.5 \mu \mathrm{g} / \mathrm{ml})$ and visualized under a UV light system (Chvallet et al, 2008).

The diagnostic procedures were evaluated and ranked on the basis of Multiattribute utility theory and Analytical hierarchy process which combine different parameters to evaluate the ranking of the diagnostic tests in any particular health care setting (MacPherson and MacQueen, 1993). The cost calculations for each method include mate- 
rial and reagent costs while the costs of equipment (microscope, ELISA reader, thermal cycler...etc.) were not included. Values were calculated in 2014 Egyptian pound. Other attributes like ease of use and interpretation and ability of batch testing were subjectively evaluated.

Statistical analysis: Data entry and analysis were performed using SPSS (Ver. 17 for Windows). The Sensitivity, specificity, the positive predictive value (PPV), the negative predictive value (NPV) and he positive and negative likelihood ratio (LR+ \& LR-) were also calculated. These parameters were calculated for each test and compared to the gold standard using MedCalc Statistical Software version 15.2.2 (using MedCalc Software bvba, Ostend, Belgium http: //www.medcalc.org 2015). The agreement between each test and the gold standard test was determined based on the calculated $\kappa$ (kappa) value gradation (Cohen, 1960). Pre-test probability and Fagan's nomograms were constructed for calculating post-test probabilities (using diagnostic test calculator at http://araw.mede.uic.edu/cgibin/testcalc.pl).

\section{Results}

A total of 100 specimens were examined by microscopy (direct wet mount and after formal ethyl-acetate concentration), Ridascreen ELISA kit and n-PCR. In comparison to Ridascreen as reference standard, the diagnostic performance and $k$ agreement of the studied methods was given.

On the basis of the estimated sensitivity and specificity, LR+ and LR- were calcu- lated for the three studied diagnostic tests. The two microscopy-based Giardia detecting tests, direct microscopy and microscopy after concentration, yielded the highest specificities $(100 \%) \& \mathrm{LR}+(\infty)$. All studied tests yielded LR-, amenable to ruling out the presence of giardiasis in patients with negative tests with $\mathrm{n}-\mathrm{PCR}$ recorded LR- (0.00).

Giardia infection was suspected with pretest probability of $19 \%$. The positive test result for microscopic examination increases the probability of giardiasis to $100 \%$ (i.e. 1:1 of cases with positive test was risky) with $95 \%$ CI 50 to $100 \%$, whereas a negative test result for microscopic examination reduces the probability to $12 \%$ (95\% CI: 9 to $16 \%$ ) for direct microscopy and $6 \%$ (95\% CI: 3 to $12 \%$ ) for microscopy after concentration. Using n-PCR as a diagnostic test for giardiasis, positive test increased probability to $74 \%$ (95\% CI: 56 to $83 \%$ ) (i.e., 1 in 1.4 of cases with positive test was risky), whereas a negative ones reduced probability to $0.0 \%$ (95\% CI: 0 to $9 \%$ ) (i.e. 1 in 1.0 of cases with negative test is well).

The multi-attribute evaluation of diagnostic performance, cost, and ease of interpretation, ability of batch testing and ability of genotyping for the different methods was given. ELISA had the highest rank (9.07) followed by n-PCR (7.53) then microscopy after formal ethyl-acetate concentration (7.40) and the last was the direct microscopy (7.25). Details were given in tables $(1,2$ $\& 3)$ and figures $(1 \& 2)$.

Table 1: Microscopy before and after concentration and n-PCR versus Ridascreen ELISA in diagnosis

\begin{tabular}{|c|c|c|c|c|}
\hline \multirow{2}{*}{ Method } & \multicolumn{2}{|c|}{ ELISA } & \\
\cline { 3 - 5 } & & Positive & Negative & Total \\
\hline \multirow{3}{*}{ Direct microscopy } & Positive & 8 & 0 & 8 \\
\cline { 2 - 5 } & Negative & 11 & 81 & 92 \\
\cline { 2 - 5 } & Total & 19 & 81 & 100 \\
\hline \multirow{3}{*}{$\begin{array}{c}\text { Microscopy after formal } \\
\text { ethyl-acetate concentration }\end{array}$} & Positive & 14 & 0 & 14 \\
\cline { 2 - 5 } & Negative & 5 & 81 & 86 \\
\hline \multirow{2}{*}{ n-PCR } & Total & 19 & 81 & 100 \\
\cline { 2 - 5 } & Positive & 19 & 7 & 26 \\
\cline { 2 - 5 } & Negative & 0 & 74 & 74 \\
\cline { 2 - 5 } & Total & 19 & 81 & 100 \\
\hline
\end{tabular}


Table 2: Diagnostic performance and kappa $(\kappa)$ agreement of used diagnostic tests

\begin{tabular}{|c|c|c|c|c|c|c|c|}
\hline Methods & $\begin{array}{c}\text { Sensitivity } \\
(95 \% \mathrm{CI})(\%)\end{array}$ & $\begin{array}{c}\text { Specificity } \\
(95 \% \mathrm{CI})(\%)\end{array}$ & $\begin{array}{c}\mathrm{PPV} \\
(95 \% \mathrm{CI})(\%)\end{array}$ & $\begin{array}{c}\mathrm{NPV} \\
(95 \% \mathrm{CI})(\%)\end{array}$ & $\begin{array}{c}\text { LR+ } \\
(95 \% \mathrm{CI})\end{array}$ & $\begin{array}{c}\text { LR- } \\
(95 \% \mathrm{CI})\end{array}$ & $\kappa$ \\
\hline Direct microscopy & $\begin{array}{c}42.11 \\
(20.25-66.5)\end{array}$ & $\begin{array}{c}100 \\
(95.55-100 \%)\end{array}$ & $\begin{array}{c}100 \\
(63.06-100)\end{array}$ & $\begin{array}{c}88.04 \\
(79.61-93.88)\end{array}$ & $\infty$ & 0.58 & 0.54 \\
$(0.39-0.85)$ & \\
\hline $\begin{array}{c}\text { Microscopy after formal } \\
\text { ethyl-acetate concentra- } \\
\text { tion }\end{array}$ & $\begin{array}{c}73.7 \\
(48.57-89.9)\end{array}$ & $\begin{array}{c}100 \\
(93.15-100 \%)\end{array}$ & $\begin{array}{c}100 \\
(73.23-100)\end{array}$ & $\begin{array}{c}94.19 \\
(86.94-98)\end{array}$ & $\infty$ & $\begin{array}{c}0.26 \\
(0.14-0.56)\end{array}$ & 0.80 \\
\hline PCR & $\begin{array}{c}100 \\
(82.35-100)\end{array}$ & $\begin{array}{c}91.36 \\
(83-96.45)\end{array}$ & $\begin{array}{c}73.08 \\
(52.21-88.43)\end{array}$ & $\begin{array}{c}100 \\
(95.14-100)\end{array}$ & $\begin{array}{c}11.57 \\
(5.7-23.49)\end{array}$ & 0.00 & 1 \\
\hline
\end{tabular}

Abbreviations: PPV, positive predictive value; NPV, negative predictive value; $\mathrm{LR}+$, positive likelihood ratio [sensitivity/(1_ specificity)]; LR-, negative likelihood ratio [(1_ sensitivity)/specificity]; CI, confidence interval. Agreement was: Poor if $k<0$; Slight if $k(0.01-0.02)$; Fair if $k(0.21-0.40)$; Moderate if $k(0.41-0.60)$; Substantial if $k(0.61-0.80)$; Almost perfect if $k(0.81-1.00)$. Priority values multiplied by ranks for each attribute for every diagnostic technique; sum up given in total.

Table 3: Multi-attribute ranking of diagnostic methods for Giardia spp.

\begin{tabular}{|c|c|c|c|c|c|c|}
\hline \multicolumn{2}{|c|}{ Evaluation item } & $\begin{array}{c}\text { Priority } \\
\text { value }\end{array}$ & $\begin{array}{c}\text { Direct } \\
\text { microscopy }\end{array}$ & $\begin{array}{c}\text { Microscopy after } \\
\text { concentration }\end{array}$ & $\begin{array}{c}\text { ELI } \\
\text { SA }\end{array}$ & $\begin{array}{c}\mathrm{n}- \\
\text { PCR }\end{array}$ \\
\hline Cost & & 0.95 & 4 & 3 & 2 & 1 \\
\hline Performance & Sensitivity & 0.35 & 1 & 2 & 3 & 4 \\
\cline { 3 - 6 } & Specificity & 0.35 & 4 & 4 & 4 & 4 \\
\hline Ease of use & & 0.9 & 1 & 2 & 3 & 1 \\
\hline Ability to perform batch testing & & 0.5 & 1 & 1 & 3 & 4 \\
\hline Ease of interpretation & & 0.15 & 2 & 1 & 3 & 4 \\
\hline Ability for species identification & & 0.07 & 0 & 0 & 1 & 4 \\
\hline \multicolumn{2}{|c|}{ Total $^{2}$} & & 7.25 & 7.40 & 9.07 & 7.53 \\
\hline
\end{tabular}

. Tests ranked from 1 to 4 for each attribute, 1 taken for least preferable characteristic and 6 for most preferred one. Every attribute prioritized by comparing its importance over other as per laboratory's infrastructure.

\section{Discussion}

Conventional microscopy proved to be the gold standard method widely used for the evaluation of patients with suspected Giardia infection. However, this method is often time-consuming and unreliable means of detection the presence of Giardia cyst. Even if the Giardia cysts present in large number verifying of their identity is difficult (Dixon et al, 1997). The low sensitivity and high incidence of false negativity of copro-diagnosis using microscopic techniques even with testing of multiple stool specimens collected over several days may limit early diagnosis and even early treatment (CLSI, 2005). This problem led to continuous trials to upgrade the existing laboratory methods to detect Giardia.

The need for a test that is easy to perform and cost effective had led to the application of immunodiagnosis for Giardia. ELISA assay has the advantage of no requiring microscopic skills. The ELISA requires less hands-on processing time (1 to $2 \mathrm{~min}$ per specimen) than microscopy (30-20 $\mathrm{min})$ (Aldeen et al, 1998). But on other side the cost of test per sample is much more than microscopic examination (El-Settawy and Fathy, 2012).

The commercially available copro-antigen detection ELISA formats use monoclonal antibodies which recognize different sets of surface epitopes. This variation in target Giardia antigens may stand behind the difference in sensitivities of the commercially available ELISA kits. Another important factor influencing the sensitivity of ELISA assay was the parasite load; Giardia cyst concentration (Vidal and Catapani, 2005). In the absence of gold standard for Giardia diagnosis, different studies suggested different standards (Van den Bossche et al, 2015). Where some studies take conventional microscopy as gold standard (Behr et $a l$, 1997) others used the direct immunoflourescence (DIF) assay as reference standard (El-Nahas et al, 2013) and even PCR assays (Hawash, 2014).

As microscopy sensitivity is influenced by the microscopes' experience and number of samples examined, in the present study ELISA assay was the reference 
standard on the basis of $98.9 \%$ sensitivity and the $100 \%$ specificity on the humans (Chakarova, 2010; El-Tantawy et al, 2012).

In the present study, ELISA assay proved better than microscopic techniques in detection of Giardia, with higher detection rate $(19 \%)$ than direct microscopy $(8 \%)$ and microscopy after concentration (14\%) in children complaining of diarrhea. Moges et al. (2010) recorded $12.6 \%$ \& $15.4 \%$ detection rates for direct Iodine and after formal-ether concentration, respectively. These results are also in agreement with findings obtained by El Safi et al. (2013) which have indicated that the immunodiagnostic methods such as ELISA are more sensitive than microscopic methods. However, ELISA test was negative for 7 samples which were positive by n-PCR. False negative results obtained by ELISA may be attributed to antigenic variability within the different isolates of Giardia and low parasite intensity (Ghaffari and Kalantari, 2014).

The excellent specificity of microscopy based tests was, however, offset by a reduced sensitivity. As the sensitivity, specificity, PPV and NPV for direct wet mount with iodine was $42.11 \%, 100 \%, 100 \%$ and $88.04 \%$, respectively. Vohra et al. (2012) also recorded $100 \%$ specificity, $100 \%$ PPV and $90.7 \%$ NPV but sensitivity was lower than that in this study ( $20 \%$ vs. $42.11 \%)$.

Comparing microscopy after formal ethylacetate concentration with ELISA showed $73.7 \%$ sensitivity, $100 \%$ specificity, with $100 \%$ PPV (no false positive samples) and 94.19\% NPV (5 false negative samples). Comparable the results $(76.9 \%$ sensitivity, $100 \%$ specificity, $100 \%$ PPV \& $72.7 \%$ NPV) were demonstrated by EL-Nahas et al. (2013) when copro-microscopy compared to direct immunofluorescence assay (DIF). Lower results were recorded by Salman (Salman, 2014) with $61.68 \%$ sensitivity, $88.37 \%$ specificity, $78.75 \%$ PPV and 88.37\% NPV. But, Van den Bossche et al. (2015) reported 90\% sensitivity and 100\% sensitivity were demonstrated.
The decreased sensitivity of copro-microscopy can be attributed to several factors and could be explained by the intermitted shedding of the parasite diagnostic stages in stool, the experience of the microscopiest and interference of anti-diarrheal drugs with the parasite.

Molecular tools including PCR assays to detect DNA of Giardia in stool have proved greater sensitivity, specificity and accuracy than the conventional diagnosis that depend on microscopy and immunoassays (El-Safi et al, 2013). Amplification of a target gene by PCR could detect single Giardia cyst (Wolfe, 1992). In this study nPCR with primers to beta giardin (bg) gene was used to detect Giardia. n-PCR detected Giardia in $26 \%$ of the examined stool specimens. In comparison to ELISA assay, nPCR showed highest sensitivity of $100 \%$ with $91.36 \%$ specificity. Giardins are defined as a family of structural proteins that are approximately $29-38 \mathrm{kDa}$ in size. The advantage of using giardin genes as targets for the molecular detection of Giardia cysts is that they are considered to be unique to Giardia and highly discriminatory, even if the sequence is relatively well conserved (Caccio, 2002).

On the other hand, the present data disagreed with Atlas (1990) who used PCR to selectively amplify the giardin gene for $\mathrm{Gi}$ ardia detection in water, where the test was not found highly sensitive. The difference might be attributed to inefficient DNA extraction and amplification of the template DNA from stool (Ghosh et al, 2000).

Likelihood ratio (LR) proved a very useful measure of diagnostic accuracy which was independent of prevalence. It directly links the pre-test and post-test probability of a disease in a specific patient (31). Good diagnostic tests have $\mathrm{LR}+>10$ and their positive result had a significant contribution to the diagnosis. In the present case, all the studied tests recorded LR+> 10 which provide convincing evidence to rule in giardiasis in most circumstances. The lower 
the LR- the more significant contribution of the test is in ruling-out, i.e. in lowering the posterior probability of the subject having the disease (Deeks and Altman, 2004).

The suspicion of the presence of a given disease was defined as pre-test probability (Watson and Petrie, 2002). It is commonly taken as the prevalence of the condition in the population. The pre-test probability of disease remains crucially important for determining the post-test probability (Deeks and Altman, 2004). Given the pre-test probability was $19 \%$, a n-PCR positive test result increases the probability of having giardiasis about 4 folds (74\%) than Ridascreen, whereas a negative n-PCR test result decreased the probability to $0 \%$.

As the sensitivity and specificity of a diagnosis should not be the only criteria in its evaluation. So, multi-attribute evaluation method was introduced. The ranked ELISA proved better than n-PCR for Giardia detection because n-PCR its wide applicability is hindered by its relative high cost and its high technology equipments. On the other hand, microscopy came last in ranking. Despite of its low cost, more time in preparation, reading and interpretation is needed and unable to genotype positive cases. MacPherson et al. (1993) also gave maximum consideration to cost effectiveness of the tests.

\section{Conclusion}

The outcome results showed a very high accuracy of n-PCR in copro-DNA for the diagnosis of giardiasis to patients at risk. The excellent sensitivity of n-PCR for copro-DNA suggests that a negative result in suspected patients should ruling out the diagnosis of giardiasis. A positive result should be interpreted in parallel with compatible clinical and microscopic findings.

Further prospective studies should focus on the quantitative PCR standardization for wide use of the test in clinical practice.

Conflict of interest: The authors have no conflict of interest with regard to the present study or its outcome data.

\section{References}

Adam, RD, 2003: Biology of Giardia. Clin. Microbiol. Rev. 4, 3:447-75.

Aldeen, W, Carroll, K, Robison, A, et al, 1998: Comparison of nine commercially available enzyme-linked immunosorbent assays for detection of Giardia lambia in fecal specimens. J. Clin. Microbiol. 36, 5:1338-40.

Atlas, RM, 1990: Environmental applications of the polymerase chain reaction. ASM News 57:630-2.

Behr, MA, Kokoskin, E, Gyorkos, TW, et al, 1997: Laboratory diagnosis for Giardia lamblia infection: A comparison of microscopy, coprodiagnosis and serology. Can. J. Infect. Dis. 8, 1:33-8.

Caccio`, SM, De Giacomo, M, Pozio, E, 2002: Sequence analysis of the b-giardin gene and development of a polymerase chain reaction-restriction fragment length polymorphism assay to genotype Giardia duodenalis cysts from human faecal samples. Int. J. Parasitol. 32:1023-30.

Chakarova, B, 2010: Comparative evaluation of the diagnostic methods for detection of $\mathrm{Gi}$ ardia intestinalis in human fecal samples. Trakia J. Sci. 8, 2:174-9.

Chvallet, M, Luche, S, Rabilloud, T, 2006: Silver staining of proteins in polyacrylamide gels. Nat. Protocol. 1, 4:1852-8.

Clinical and Laboratory Standard Institute (CLSI), 2005: Procedures for the Recovery and Identification of Parasites from the Intestinal Tract; Approved Guidelines. $2^{\text {nd }}$ ed.; CLSI Document M28-A2.

Cohen, J, 1960: A coefficient of agreement for nominal scales. Educ. Psychol. Meas. 20:37-46. Deeks, JJ, Altman, DG, 2004: Diagnostic tests 4: Likelihood ratios. BMJ 229, 7458:168-9.

Dixon, BR, Parenteau, M, Martineau, $C$, et al, 1997: A comparison of conventional microscopy, immunofluorescence microscopy and flow cytometry in the detection of Giardia lamblia cysts in beaver fecal samples. J. Immunol. Meth. 202:27-33.

El-Badry, AA, Al-Ali, KH, Mahrous, AS, 2010: Molecular identification \& prevalence of Giardia lamblia \& Cryptosporidium in duodenal aspirate in Al-Madinah. J. Med. Biomedical Sci. 1, 2:47-52.

El-Nahas, HA, Salem, DA, El-Henawy, AA, El-Nimr, HI, Abdel-Ghaffar, HA, et al, 2013: Giardia diagnostic methods in human fe- 
cal samples: A Comparative Study. Cytometry Part B. 84:44-9.

El-Safi, SH, Al-Maqati, TN, Hussein, MI, et al, 2013: Comparison of microscopy, rapid immunoassay, and molecular techniques for detection of Giardia lamblia and Cryptosporidium parvum. Parastol. Res. 112:1641-6.

El-Safi, SH, Al-Maqati, TN, Hussein, MI, et al, 2013: Comparison of microscopy, rapid immunoassay, and molecular techniques for detection of Giardia lamblia and Cryptosporidium parvum. Parasitol. Res. 112:1641-6.

El-Settawy, MA, Fathy, GM, 2012: Evaluation and comparison of PCR coproantigen ELISA and microscopy for diagnosis of Cryptosporidium in human diarrhoeic specimens. J. Am. Sci. 8, 12:1378-85.

El-Tantawy, NL, El-Nahas, HA, Sultan, DM, 2012: Diagnosis of Giardia and Cryptosporidium infection in children with diarrheal illness using rapid immunochromatographic. Arch. Clin. Microbiol. 3, 1:1-5.

Garcia, LS, 2007: Diagnostic Medical Parasitology, $5^{\text {th }}$ ed. ASM Press, Washington D.C.

Garcia, LS, Shimizu, RY, 1997: Evaluation of nine immunoassay kits (enzyme immunoassay and direct fluorescence) for detection of Giardia lamblia and Cryptosporidium parvum in human fecal specimens. J. Clin. Microbiol. 35: 1526-9.

Ghaffari, S, Kalantari, N, 2014: Recognition of Cryptosporidium oocyts in fresh and old stool samples: comparison of four techniques. Asian Pac. J. Trop. Biomed. 4, 2:S570-4.

Ghosh, S, Debnath, A, Sil, A, et al, 2000: PCR detection of Giardia lamblia in stool: targeting intergenic spacer region of multicopy rRNA gene. Mol. Cell. Probes 14, 181-189.

Hawash, Y, 2014: Evaluation of an immunoassay-based algorithm for screening and identification of Giardia and Cryptosporidium antigens in human fecal specimens from Saudi Arabia. J. Parasitol. Res. Article ID 213745, doi: 10.1155/2014/213745.

Johnston, SP, Ballard, MM, Beach, MJ, et al, 2003: Evaluation of three commercial as- says for detection of Giardia and cryptosporidium organisms in fecal specimens. J. Clin. Microbiol. 41, 2:623-6.

Knottnerus, JA, van Weel, C, Muris, JWM, 2002: Evidence base of clinical diagnosis: Evaluation of diagnostic procedures. Br. Med. J. 324:477-80.

MacPherson, D, MacQueen, R, 1993: Cryptosporidiosis multi-attribute evaluation of six diagnostic methods. J. Clin. Microbiol. 3:198202.

Moges, F, Belyhun, Y, Tiruneh, M, et al, 2010: Comparison of formol-acetone concentration method with that of the direct iodine preparation and formol-ether concentration methods for examination of stool parasites. Ethiop. J. Hlth. Dev. 24, 2:148-51.

Salman, YJ, 2014: Efficacy of some laboratory methods in detecting Giardia lamblia and Cryptosporidium parvum in stool samples. Kirkuk Uni. J. Sci. Studies 9, 1:7-17.

Savioli, L, Smith, H, Thompson, A, 2006: Giardia \& Cryptosporidium join the neglected disease Initiative. Trends Parasitol. 22:203-8.

Van den, Bossche, D, Cnops, L, Verschueren, J, et al, 2015: Comparison of four rapid diagnostic tests, ELISA, microscopy and PCR for the detection of Giardia lamblia, Cryptosporidium spp. and Entamoeba histolytica in feces. J. Microbiol. Meth. 110:78-84.

Vidal, AMB, Catapani, WR, 2005: Enzymelinked immunosorbent assay (ELISA) immunoassaying versus microscopy: advantages and drawbacks for diagnosing giardiasis. Sao-Paulo Med. J. 123, 6:282-5.

Vohra, P, Singla, P, Sharma, M, et al, 2012: Comparison of direct immunofluorescence, iodine-saline wet mount and modified acid fast staining methods for detection of Cryptosporidium and Giardia spp. in human fecal specimens. J. Evol. Med. Dental Sci. 1, 4:285-90.

Watson, PF, Petrie, A, 2010: Method agreement analysis: A review of correct methodology. Theriogenology 73:1167-79.

Wolfe, MS, 1992: Giardiasis. Clin. Microbiol. Rev. 5, 93-100.

\section{Explanation of Figures}

Fig 1: Representative ethidium bromide-stained $1.5 \%$ agarose gel picture showing amplification products $(\approx 511 \mathrm{bp})$ of $\mathrm{n}$ PCR targeting beta giardin (bg) gene of Giardia. L lane for 100bp LADDER; lane 1 is negative control; lane 2 positive control; lane 5, 9, 10, 11 positive specimens; lane 3, 4, 6, 7, 8, 12, 13 negative.

Fig 2: Fagan's nomogram for calculating post-test probability of giardiasis for n-PCR compared to microscopic methods (Direct and after formal-ethyl acetate concentration). Lines were drawn from pre-test probability on left through likelihood ratios (LR+ \& LR-) in middle and extended to posterior probabilities on right. Positive test result (upper blue line) and negative test result (lower red line) 

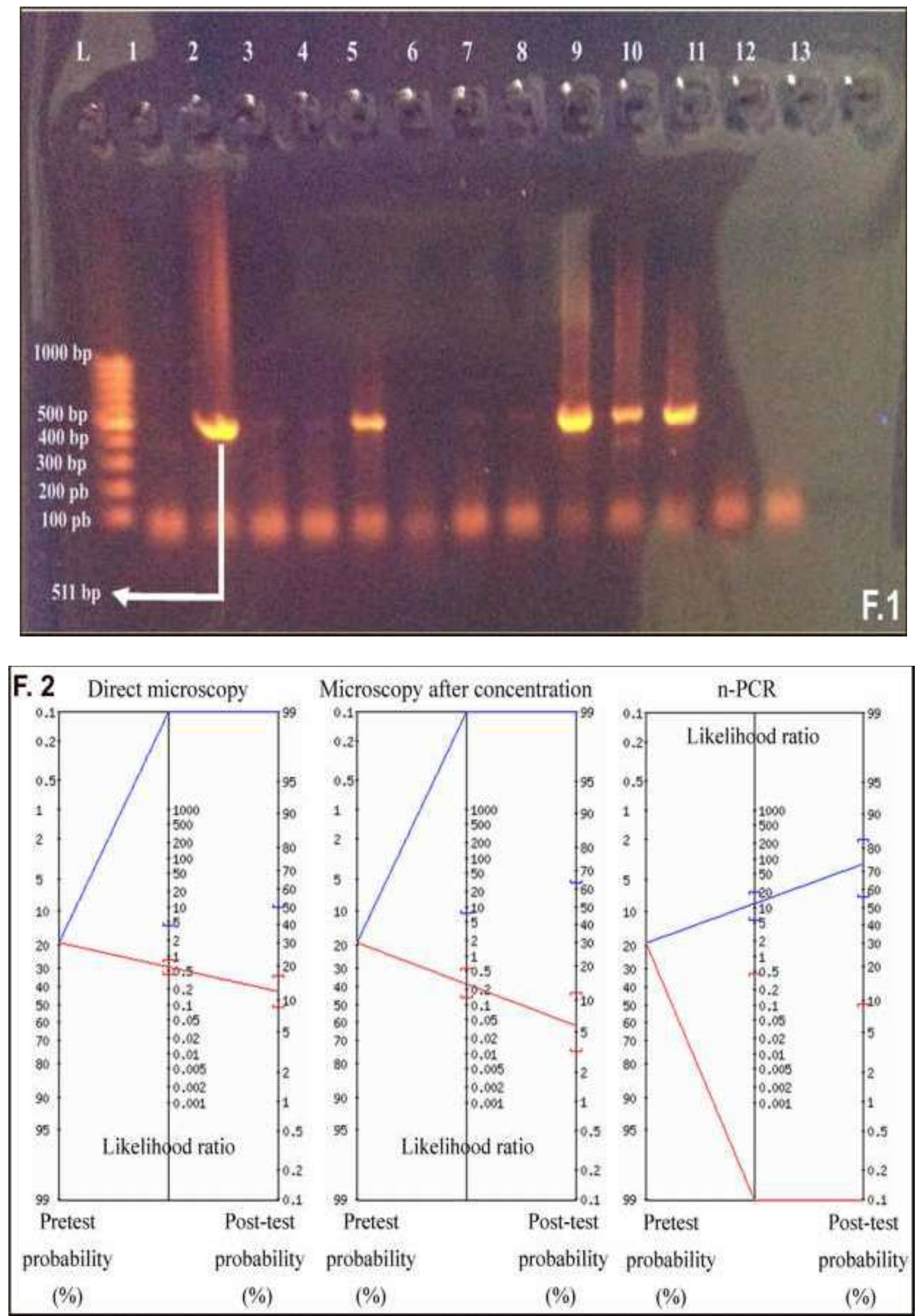